\title{
The nature of long-GRB host galaxies from chemical abundances
}

\author{
X. L. Fan ${ }^{1,2}$, J. Yin ${ }^{3,1}$, and F. Matteucci ${ }^{1,2}$ \\ 1 Dipartimento di Fisica, Sezione di Astronomia, Università di Trieste, via G.B. Tiepolo 11, 34131 Trieste, Italy \\ e-mail: fan@oats.inaf.it \\ 2 INAF Osservatorio Astronomico di Trieste, via G.B. Tiepolo 11, 34131 Trieste, Italy \\ 3 Key Laboratory for Research in Galaxies and Cosmology, Shanghai Astronomical Observatory, Chinese Academy of Sciences, \\ 80 Nandan Road, Shanghai 200030, PR China
}

Received 29 June 2010 / Accepted 23 July 2010

\begin{abstract}
Context. Gamma-ray bursts (GRBs) were the most energetic events after the Big Bang and they have been observed up to very high redshift. Measurements of the chemical abundances are now available for the galaxies hosting such events, which are assumed to originate from the explosion of very powerful supernovae (Type $\mathrm{Ib} / \mathrm{c}$ ), and provide the opportunity to study the nature of these host galaxies.

Aims. We identify the hosts of long GRBs (LGRBs) observed at both low and high redshift to determine whether the hosts are galaxies of the same type at different cosmic epochs.

Methods. We adopt detailed chemical evolution models for galaxies of different morphological type (ellipticals, spirals, irregulars) that follow the time evolution of the abundances of several chemical elements ( $\mathrm{H}, \mathrm{He}, \alpha$-elements, $\mathrm{Fe}$ ), and compare the results with the observed abundances and abundance ratios in galaxies hosting LGRBs.

Results. We find that the abundances and abundance ratios predicted by models devised for typical irregular galaxies can reproduce the abundances of the hosts at both high and low redshift. We also find that the predicted Type Ib/c supernova rate for irregulars is in good agreement with observations. Models for spirals and particularly ellipticals fit neither the high-redshift hosts of LGRBs (DLA systems) nor the low redshift hosts: in particular, ellipticals cannot possibly be the hosts of gamma-ray bursts at low redshift since they exhibit little star formation, hence no supernovae $\mathrm{Ib} / \mathrm{c}$.

Conclusions. We conclude that the observed abundance and abundance ratios in LGRBs hosts suggest that these hosts are irregular galaxies at both high and low redshift, thus demonstrating that the host galaxies are the same type of galaxies observed at different ages.
\end{abstract}

Key words. galaxies: high-redshift - Galaxy: abundances - galaxies: ISM - gamma rays: galaxies - gamma rays: ISM

\section{Introduction}

Gamma-ray bursts (GRBs) were the most energetic events after the Big Bang. They can be detected back to the onset of reionization (e.g., Greiner et al. 2009; Salvaterra et al. 2009; Tanvir et al. 2009) due to their brightness in the first few hours after the explosion (Lamb \& Reichart 2000). Long GRBs (LGRBs) $\left(T_{90} \gtrsim 2\right)$ are transient sources followed by long-lasting afterglows emitting energy intensely across the full range of the electromagnetic spectrum. They are effective and informative probes of many aspects of astrophysics, such as cosmic starformation rate (SFR) (Kistler et al. 2009), cosmic dust (Liang \& Li 2009), galaxy properties (Castro Cerón et al. 2010), and interstellar medium (ISM) properties (Levesque et al. 2010a). Whether the properties of LGRB progenitors depend or not on metallicity is a key issue in understanding the observations. Low metallicity is indicative of a less evolved galaxy, usually of low stellar mass, high gas-to-stellar mass ratio, and low luminosity. LGRB progenitors are understood to be low-metallicity massive core-collapse stars (Woosley 1993; Woosley \& Heger 2006a; Yoon et al. 2008) observed in association with supernovae ( $\mathrm{SNe}$ ) $\mathrm{Ib} / \mathrm{c}$, which in turn originate in massive stars and therefore reside in active star-forming regions (e.g. Galama et al. 1998; Hjorth et al. 2003; Pian et al. 2006; Woosley \& Heger 2006b).
However, this low-metallicity progenitor channel is challenged by so-called "dark" LGRB (LGRB051022 and LGRB020819) associated with a high-metallicity environment (Graham et al. 2009; Levesque et al. 2010b).

Different systems are selected by different methods from high to low redshift. Two kinds of LGRB associated systems are selected: LGRB host galaxies at low redshift and LGRBassociated damped Lyman-alpha systems (LGRB-DLAs) at high redshift. LGRB host galaxies have been studied by several authors using observational data (e.g. Chary et al. 2007; Michałowski et al. 2008; Castro Cerón et al. 2010; Levesque et al. 2010a; Savaglio et al. 2009; Wolf \& Podsiadlowski 2007, among others) and simulations (e.g. Mao 2010; Chisari et al. 2010; Campisi et al. 2009; Calura et al. 2009; Lapi et al. 2008; Nuza et al. 2007, among others). All these results find that at low redshift $(z<2)$, the typical hosts of LGRB are small, starforming, low-metallicity irregular galaxies.

At high redshift $(z>2)$, as for QSO-DLA systems $\left(\log N_{\mathrm{HI}}>\right.$ $20 \mathrm{~cm}^{-2}$ ), the LGRB-DLAs are observed (e.g. Jakobsson et al. 2006; Prochaska et al. 2007a; Fynbo et al. 2009). The nature of DLAs remains a matter of debate. The DLAs in QSO spectra are assumed to form in the ISM of galaxies located in front of the QSO. The high luminosity of the QSOs usually makes it difficult detect the DLA galaxies directly. The hypothesis that 
these DLAs are the progenitors of present disk galaxies (Wolfe et al. 1986; Naab \& Ostriker 2006) is challenged by the low metallicity $\left(\simeq 1 / 100 Z_{\odot}\right)$ of DLAs and the flat age-metallicity relation of disk stars (the disk is rapidly enriched to $\simeq 1 / 3 Z_{\odot}$ ) (Akerman et al. 2004; Pettini 2006). Using detailed chemical evolution models, Calura et al. (2003, 2009) investigated the nature of DLAs and suggested that the majority of them, including the LGRB-DLAs, may be either spiral disks observed at large galactocentric distances, irregular galaxies such as the LMC, or starburst dwarf irregulars observed at different times after the last burst of star formation. On the other hand, massive elliptical galaxies are unlikely to be DLA systems even at very high redshift owing to their intensive quick enrichment, which produces high $[\alpha / \mathrm{Fe}]$ ratios, that is inconsistent with the data observed for DLAs. However, among the identified high redshift DLA host galaxies, one DLA host galaxy is a luminous Lyman-break galaxy (DLA 2206-19A, Møller et al. 2002). By comparing the luminosity functions of DLAs and LBGs, Wolfe (2005) concluded that there is a significant overlap between the DLA and LBG populations. Fynbo et al. (2008) compared the metallicity distributions of LGRB-DLAs and LBGs. Their results support the hypothesis that LGRB-DLAs could arise from a population of LBGs that are not heavily obscured. LBGs are understood to be small young star-forming ellipticals (Matteucci \& Pipino 2002; Pipino et al. 2010). Additionally, Zwaan et al. (2005) showed that in the local universe the luminosity distribution of galaxies producing DLAs is nearly flat from $M_{B} \approx-20$ to $M_{B} \approx-15$, which implies that the DLA host galaxies should belong to a complex population (a single type population could not span such a wide luminosity range). it is normally unknown in which region of the galaxy the line of sight from the QSO and LGRBs intersect the ISM of the galaxy. Prochaska et al. (2007b) argued that LGRB-DLAs preferentially probes denser, more depleted, higher metallicity gas located in the inner few kpc of the ISM compared to QSO-DLAs. This idea is supported by the distribution of HI column densities for GRB absorbers produced using a high resolution simulation of galaxy formation (Pontzen et al. 2010). In principle, it should be possible for high-redshift LGRB-DLAs to be a phase of low-mass star-forming ellipticals, which is what we test in this paper.

In this article, we use the most recently observed abundances of the LGRB-associated systems, LGRB-DLAs at high redshift and LGRB host galaxies at low redshift, to try to determine: i) which galaxies are the hosts of LGRBs? ii) whether the LGRB-associated systems represent an evolutionary sequence, i.e., whether the same objects at low and high redshift are just seen at different phases of their evolution? To do this, we adopt updated chemical evolution models for galaxies of different morphological type that reproduce the properties of galaxies (Pipino et al. (2010) for ellipticals, Yin et al. (2010b) for irregulars and spirals), and compare our predicted chemical abundances with the observed ones.

The paper is organized as follows: in Sect. 2, we describe our galaxy formation scenario; in Sect. 3 we summarize the observational constraints; our results and discussions are presented in Sect. 4; and our summary and conclusions are drawn in Sect. 5. Throughout the paper, we adopt a $(0.7,0.3,0.7)$ cosmology.

\section{Galaxy formation scenario}

We now summarize our adopted galaxy formation models for galaxies of different morphological type. We direct the reader to Pipino et al. (2010) (ellipticals) and Yin et al. (2010b) (irregular and spirals) for equations and related details. Galaxies of different morphological type have different star formation histories (Matteucci 2001). Different star formation histories produce different abundance ratios, particularly in terms of $[\alpha / \mathrm{Fe}]$ as a function of $[\mathrm{Fe} / \mathrm{H}]$, while other properties may not show such large differences, such as metallicity and stellar mass at high and low redshift, respectively (see below).

\subsection{Ellipticals}

The elliptical galaxies are assumed to evolve following an instantaneous mixing of gas but not according to the instantaneous recycling approximation, i.e., we take into account stellar lifetimes. The model we adopt is similar to that described in Pipino et al. (2010) except that we do not consider the dust production and evolution since here we compare our results only with extinction-corrected data (Savaglio 2006; Savaglio et al. 2009; Levesque et al. 2010a), assuming that their dereddenings are reliable (but see Li et al. 2008). The initial conditions for ellipticals allow formation by either collapse of a gas cloud into the potential well of a dark matter halo or, more realistically, the merging of several gas clouds. In any case, the timescale for both processes, $\tau$, should be short $(<0.5 \mathrm{Gyr})$, so that the ellipticals form in a short time. The rapid collapse triggers an intense and rapid star-formation process, which can be considered as a starburst that lasts until a galactic wind, powered by the thermal energy injected by stellar winds and SN (Ia, Ibc, II) explosions, occurs. At that time, the thermal energy is equivalent to the binding energy of gas, and all the residual gas is assumed to be lost. Numerical simulations have demonstrated that a reliable assumption is that no more than $20 \%$ of the total blast-wave energy of supernovae should be used to thermalize the gas (Pipino \& Matteucci 2004). The galactic wind develops outside-in, and after the wind the star formation stops. Therefore, in this picture the outer regions of ellipticals cease the star formation before the inner regions. After that time, the galaxies evolve passively. An important assumption is that the efficiency of star formation, namely the SFR per unit mass of gas, increases with galactic mass: this scenario can explain the chemical downsizing (for this definition of downsizing, see Cowie et al. 1996), namely that more massive ellipticals have larger $[\alpha / \mathrm{Fe}]$ ratios in their dominant stellar populations than smaller ellipticals, as shown by Pipino \& Matteucci (2004). In principle, to explain the increase in the $[\alpha / \mathrm{Fe}]$ ratio one could assume a variable initial mass function (IMF) becoming flatter with increasing galactic mass, or a dry-merger scenario (Bell et al. 2006), but in both of these cases other observations are violated. For instance, a top-heavy IMF is inconsistent with the mass-luminosity relationship (Padovani \& Matteucci 1993; Matteucci \& Padovani 1993), while the drymerger scenario can reproduce the downsizing in $[\mathrm{Mg} / \mathrm{Fe}]$-mass relation but not the mass-metallicity relation and viceversa (see Pipino \& Matteucci 2008). One can certainly propose a selective metal loss (e.g. Fe should be lost more easily than $\mathrm{Mg}$ in the more massive galaxies) mechanism in any scenario to fit this chemical downsizing, but this assumption has no a physical basis (see Matteucci et al. 1998). In this paper, we adopt a Salpeter (1955) one-slope IMF and the same stellar yields as in Pipino et al. (2010). In particular, for massive stars we adopt the yields suggested by François et al. (2004), for the low and intermediate mass stars the yields of van den Hoek \& Groenewegen (1997), and for SNe Ia the yields of Iwamoto et al. (1999) (model W7). Finally, the assumed SFR is a Schmidt (1959) law that is linearly dependent on the gas density. 


\subsection{Spirals and irregulars}

Spirals and irregulars are galaxies harboring either recent or active star formation activity. At variance with elliptical galaxies, a slow and continuous star formation regime is assumed in spirals and irregulars. In the irregular galaxies, the efficiency of star formation is assumed to be lower than in spirals, an assumption that has been proven to be correct.

Following the work of Yin et al. (2010a,b), we build a onezone model for irregulars, and assume that the galaxy is built up by continuous infall of primordial gas until a given mass is accumulated. For the spiral disks, we develop the same model as for irregulars but with higher star formation efficiency (SFE) and higher luminous mass. As suggested by Calura \& Matteucci (2006), the main properties of local galaxies of different morphological type could be reproduced mainly by decreasing the star formation efficiency from early to late types. Therefore, we assume for spirals and irregulars a continuous SFR with SFE lower than the one used for ellipticals $\left(\simeq 3-22 \mathrm{Gyr}^{-1}\right)$, e.g. $\simeq 1 \mathrm{Gyr}^{-1}$ for spirals and $\simeq 0.1-0.05 \mathrm{Gyr}^{-1}$ for irregulars. The irregulars are assumed to assemble by infall of gas on a relatively short timescale ( $\tau \sim 1 \mathrm{Gyr})$ and the infall mass is assumed to be $5 \times 10^{9} M_{\odot}$ for all models, whereas the spiral disks are proposed to form by means of a slower accreting process $(\tau \sim 7 \mathrm{Gyr})$ and the total infall mass is $10^{11} M_{\odot}$. Galactic winds triggered by SNe are also considered in the same way as for ellipticals. According to the different SFE in each model, and consequently different $\mathrm{SN}$ feedback, the galactic wind develops at different times in different models, which then end up with different final stellar masses.

Therefore, the main difference between models for galaxies of different morphological type in is the efficiency star formation, which is higher in more massive, early-type objects, and consequently whether a galactic wind occurs and when.

\section{LGRB data}

The high-redshift LGRB-DLA data are inferred from the restframe ultraviolet (UV) absorption lines. On the other hand, lowredshift LGRB host-galaxy data are measured using the restframe optical emission lines of $\mathrm{H}$ II regions. The absorption lines in the UV and emission lines in the optical band have little overlap in terms of wavelength, making the detection of LGRBDLAs and LGRB host galaxies for the same target particularly hard. In this section, we briefly summarize the observations used in this paper.

\subsection{Low redshift case: LGRB host galaxies}

The properties of GRB host galaxies were studied by Savaglio et al. (2009) using the largest sample studied to date (40 LGRBs in total $46 \mathrm{GRBs})$. Owing to the limitation of measurements, $89 \%$ of the hosts are at $z \leq 1.6$. In addition, all 14 LGRBs considered in this paper for which $[\mathrm{O} / \mathrm{H}]$ data are available, are at $z<1$. Ten of them are studied with more detailed optical emission-line diagnostics by Levesque et al. (2010a).

\subsubsection{Morphology}

The typical morphology of LGRB host galaxies remains unclear. The typical observed hosts of LGRB are small, star-forming, low-metallicity galaxies. But since there is no reason for LGRBs to assume any particular galactic morphology, they should be, in principle, observed in galaxies of all morphological types. However, by comparing the most faint observed star-forming
LGRB host galaxies and the rapid evolution of elliptical galaxies, we can deduce that any elliptical hosting a low-redshift $(z \leq 2)$ LGRBs should be small $\left(\sim 10^{10} M_{\odot}\right)$ and in its very early phase of formation. This formation scenario is consistent with the model of Pipino et al. (2010), where we suggested that the QSO hosts $\left(\sim 10^{12} M_{\odot}\right)$ formed at $z \sim 8$ and the LBGs $\left(\sim 5 \times 10^{10} M_{\odot}\right)$ formed at $z \leq 4$ implying that at higher redshift $(z>1)$ one could observe more massive LGRB host elliptical galaxies. If this scenario were correct, we would observe a mass-redshift relation for LGRB hosts.

\subsubsection{Star formation rates}

The SFRs are inferred from either the rest-frame UV $(\lambda=$ $2800 \AA$ ) continuum luminosity, $\mathrm{H} \alpha$ flux, or other SFR calibrations (see data references for details). The measured SFRs of the adopted samples span from 0.05 to $36.46 M_{\odot} \mathrm{yr}^{-1}$ (Savaglio et al. 2009). Given the complex and uncertain SFR measurements, here we adopt only the critical star-forming criterion, namely that $S F R>0$, for the LGRB host galaxies.

\subsubsection{Stellar mass}

The stellar mass can be estimated from observed multi-band optical- near-infrared (NIR) photometry by performing spectral energy distribution (SED) fitting ${ }^{1}$ (Savaglio et al. 2009). Some authors (e.g. Castro Cerón et al. 2010; Svensson et al. 2010) derived the stellar mass only using the rest-frame $K$-band flux density assuming that it is a reliable estimator. Alternatively, the stellar mass can be estimated using an empirical relation, when other parameters are available (Savaglio 2006). The Savaglio et al. (2009) results indicate that the stellar mass of LGRB host galaxies are small (maximum $M_{\star}<10^{11} M_{\odot}$ ). Because of the faintness of LGRB host galaxies, and the average observed stellar mass $\left(\left\langle M_{\star}\right\rangle=10^{9.3} M_{\odot}\right)$ lower than field galaxies (Savaglio et al. 2009), we adopt $5 \times 10^{9} M_{\odot}$ as the typical infall mass of our fiducial model for irregulars. On the other hand, we consider low mass elliptical galaxies and adopt $10^{10} M_{\odot}$ as the typical mass of our fiducial model of low-mass ellipticals.

\subsubsection{Metallicity}

The metallicity (expressed as $\log (\mathrm{O} / \mathrm{H})+12$ ) was inferred from extinction-corrected emission-line fluxes using $R_{23}$, O3N2-metallicity relation, and auroral [O III] $\lambda 4363$ diagnostics by Savaglio et al. (2009) and Levesque et al. (2010a). The majority of the measured oxygen abundances are subsolar. However, two super-solar host galaxies are reported (Levesque et al. 2010b; Graham et al. 2009). Those super-solar metallicities challenge the assumption of a low metallicity threshold for LGRB progenitors. As we later show, their hosts may be described by our spiral/irregular model with $S F E \sim 0.1$ (see Fig. 2). In this case, the hosts may be the disks of spirals.

\subsection{High redshift case: LGRB-DLAs}

The high-redshift GRB-DLA data are measured by the restframe UV absorption lines detected in the optical afterglow

\footnotetext{
1 The mass fitting is robust (Shapley et al. 2005), despite the metallicity, dust extinction, and age being degenerate (see Chen et al. 2010, who compared six evolutionary population synthesis (EPS) models and concluded that different EPS models are necessary to reproduce different stellar populations).
} 
Table 1. Model predictions at 13 Gyr.

\begin{tabular}{cccccc}
\hline \hline Mod & infall mass $\left(M_{\odot}\right)$ & $S F E\left(\mathrm{Gyr}^{-1}\right)$ & Gas mass $\left(10^{8} M_{\odot}\right)$ & Stellar mass $\left(10^{9} M_{\odot}\right)$ & $S F R\left(M_{\odot} \mathrm{yr}^{-1}\right)$ \\
\hline 1 & $5 \times 10^{9}$ & 1 & 0.079 & 2.65 & 0.0079 \\
2 & $5 \times 10^{9}$ & 0.1 & 0.827 & 1.34 & 0.0083 \\
3 & $5 \times 10^{9}$ & 0.05 & 7.34 & 1.12 & 0.037 \\
4 & $5 \times 10^{9}$ & 0.01 & 46.25 & 0.30 & 0.046 \\
5 & $10^{11}$ & 1 & 31.25 & 68.5 & 31 \\
6 & $10^{10}$ & 3 & 0.0 & 4.09 & 0 \\
7 & $10^{12}$ & 22 & 0.0 & 530 & 0 \\
\hline
\end{tabular}

spectra. Using the rest-frame UV absorption-line column densities, one measures the cold gas-phase ( $T \leq 1000 \mathrm{~K})$ abundance, which is valid if we assume negligible ionization corrections and that the dust extinction corrections were appropriate. The observed abundances (e.g. Si, S, Fe) in LGRB-DLA are subsolar, spanning from 1/100 to near solar (Savaglio 2006; Prochaska et al. 2007b; Savaglio et al. 2009). Since Prochaska et al. (2007b) did not take into account the dust-depletion correction, we adopted the data in Savaglio (2006).

In the literature (e.g. Savaglio 2006), by adopting one reliable element abundance tracer and available dust-depletion corrections, the metallicity is generally given by that particular element abundance. Since the elements are produced by different stars with different lifetimes, the metallicity estimated by those elements cannot represent the true metallicity (which is normally estimated by $[\mathrm{O} / \mathrm{H}]$ ). Measuring LGRB-DLA metallicity is a challenge at $z<1.6$, since the Ly $\alpha$ absorption line is in the UV. Rau et al. (2010) presented an extremely metal-poor LGRBDLA system $\left(Z \sim 1 / 300 Z_{\odot}\right)$. They measured several chemical abundances and suggested that the apparent conflict between the high $[\mathrm{Si} / \mathrm{Fe}]$ and low $[\mathrm{O} / \mathrm{Fe}]$ ratios found in this object is caused by the underestimate of the $\mathrm{O}$ column density.

\section{Results and discussion}

The nature of DLAs remains unclear, but many authors have suggested that their chemical properties and star-formation histories can be reproduced by models of irregular galaxies that slowly evolve due to a mild SFR (Bradamante et al. 1998; Romano et al. 2006; Yin et al. 2010b). Typical elliptical galaxies are instead very unlikely to host the DLAs observed at high redshift primarily because ellipticals form rapidly (in less than $1.5 \mathrm{Gyr}$, see Pipino \& Matteucci 2004; Pipino et al. 2010) consuming and then losing any residual gas so quickly that additional star formation is inhibited. Therefore, to observe an elliptical when it is still forming stars, one should observe it at very high redshift, higher than the typical redshift of DLAs. Moreover, if the DLAs represent an early phase of galaxy formation, regardless of their morphological type, we should observe DLAs with different properties (e.g. different $[\alpha / \mathrm{Fe}]$ versus $[\mathrm{Fe} / \mathrm{H}]$ relations), which reflect the different morphological galaxy evolution history, and this is not the case.

To verify what has been stated above, we computed chemical evolution models for galaxies of different morphological type. In Table 1, we summarize some model results: in the first column we present the model identification, in the second column the total infall mass, in the third column the assumed SFE, in the fourth column the predicted present-time gas mass, in the fifth column the predicted present-time stellar mass, and in the sixth column the predicted present-time (13.7 Gyr) SFR expressed in $M_{\odot} \mathrm{yr}^{-1}$. Models from 1 to 4 refer to dwarf irregulars and all assume an infall mass of $5 \times 10^{9} M_{\odot}$, whereas model 5 refers to

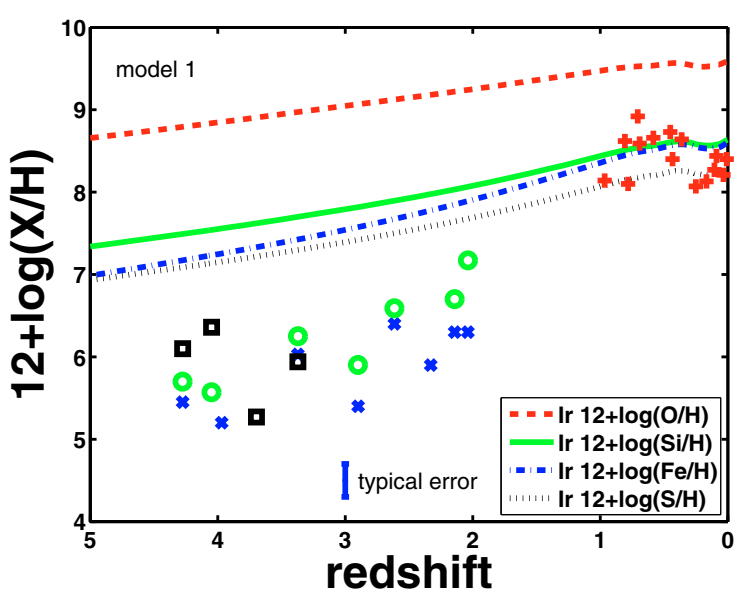

Fig. 1. $12+\log (\mathrm{X} / \mathrm{H})$ as a function of redshift. Predicted lines are from the irregular galaxy model with $S F E=1$, as indicated in the figure. High redshift $(z>2)$ data are LGRB-DLA systems and low redshift $(z<1)$ data are LGRB host galaxies. Red pluses, green circles, blue crosses, and black squares are relative to $\mathrm{O}, \mathrm{Si}, \mathrm{Fe}$, and $\mathrm{S}$ abundances, respectively. Typical error in data is shown by a vertical line. Data are derived from Savaglio (2006), Levesque et al. (2010a), Savaglio et al. (2009), and Rau et al. (2010).

an infall mass of $10^{11} M_{\odot}$ and the disk of a spiral. Model 6 represents a small elliptical with infall mass $\sim 10^{10} M_{\odot}$ and model 7 a massive elliptical with infall mass of $10^{12} M_{\odot}$. We note that the predicted SFRs for dwarf irregulars agree with the lowest values estimated by Savaglio et al. (2009), while the SFR for the spiral disk agrees with the highest observed values, and the gas masses agree with the values measured for dwarf irregulars. No star formation occurs in the elliptical model after the galactic wind (occurring before $1.5 \mathrm{Gyr}$ since the beginning of star formation), which implies that ellipticals cannot be the observed nearby $(z<1)$ host galaxies of LGRBs, unless they just formed. On the other hand, the outermost regions of galactic disks could be associated with DLAs simply because their chemical and gaseous properties resemble those of irregular galaxies, as shown in previous works.

\subsection{Metallicity-redshift relation for LGRB-associated systems}

To establish which galaxies host the LGRBs at both low and high redshift, we compare our model results with the observed abundances in LGRB-associated systems (see Figs. 1-4). The model with $S F E=1.0$ simulates the evolution of a small disk of either a spiral galaxy or an irregular galaxy undergoing more intense star formation than the average, whereas the $S F E=0.1-0.05$ is typical of dwarf irregular galaxies (see Romano et al. 2000; 


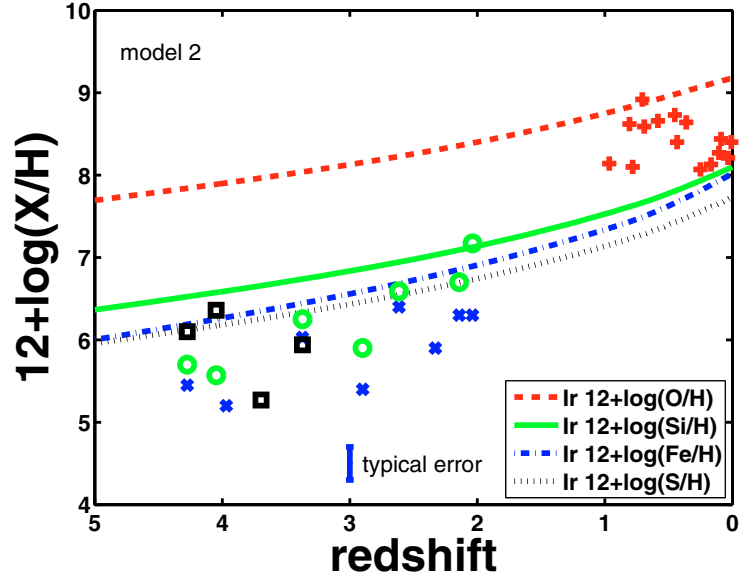

Fig. 2. $12+\log (\mathrm{X} / \mathrm{H})$ as a function of redshift. The same as Fig. 1 but for $S F E=0.1$

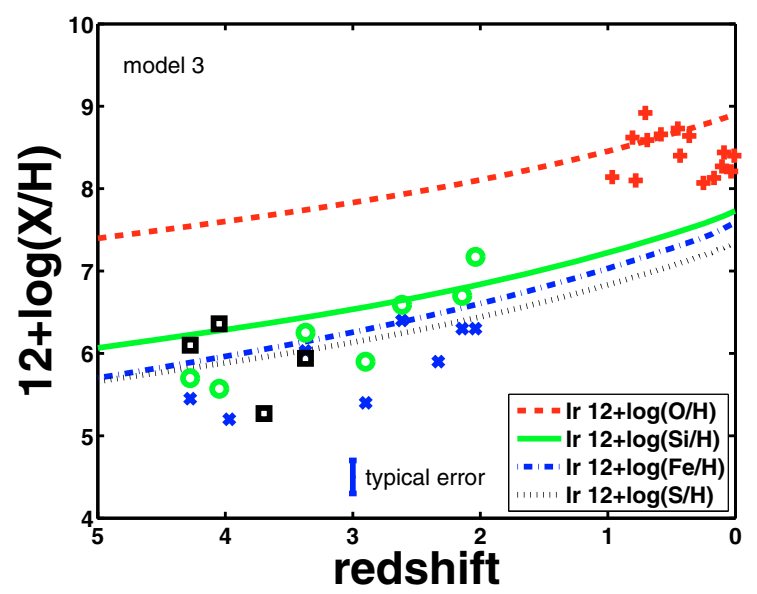

Fig. 3. $12+\log (\mathrm{X} / \mathrm{H})$ as a function of redshift. The same as Fig. 1 but for $S F E=0.05$.

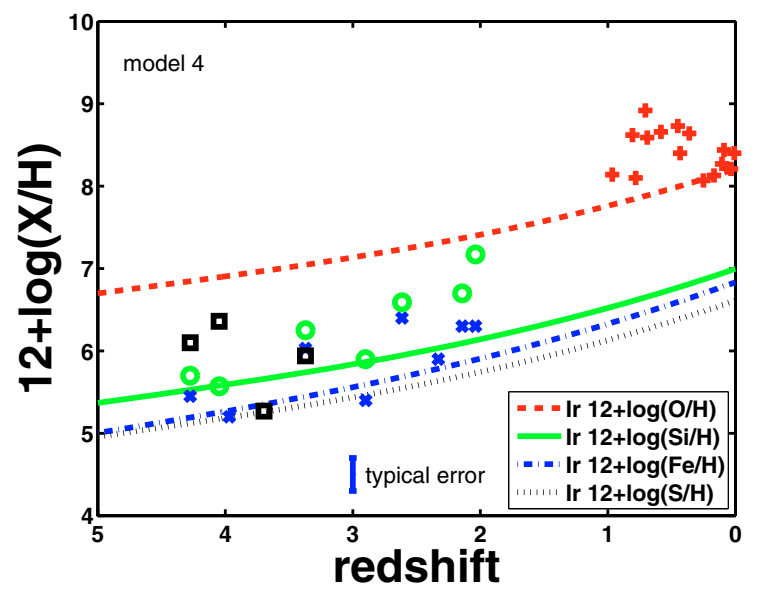

Fig. 4. $12+\log (\mathrm{X} / \mathrm{H})$ as a function of redshift. The same as Fig. 1 but for $S F E=0.01$.

Lanfranchi \& Matteucci 2003). From Figs. 1-4, one can see that the value $S F E=1.0$ repoduces the LGRB-associated systems at neither high nor low redshift, whereas the model for irregulars galaxies with $S F E=0.1-0.05$ can closely fit all the data. On the other hand, values of $S F E<0.05$ do not fit the data. This comparison clearly indicates that the associated systems of the

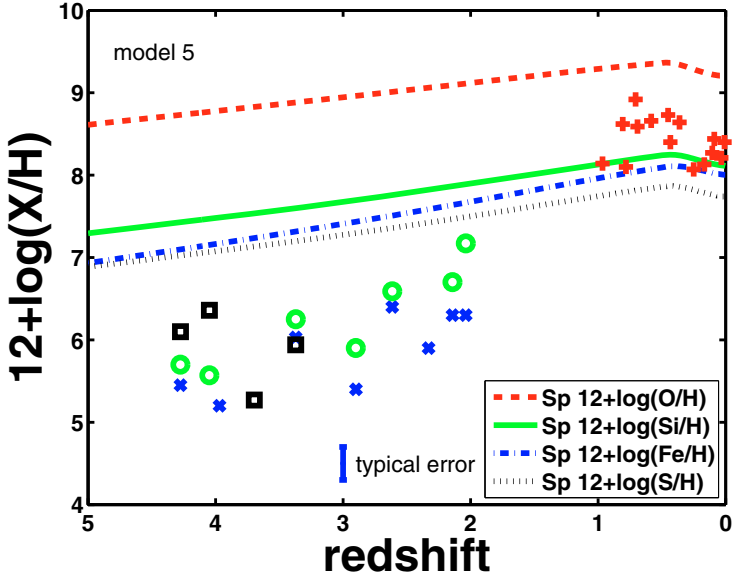

Fig. 5. $12+\log (\mathrm{X} / \mathrm{H})$ as a function of redshift. The same as Fig. 1 but for the spiral galaxy model with $S F E=1$ and infall mass $10^{11} M_{\odot}$.

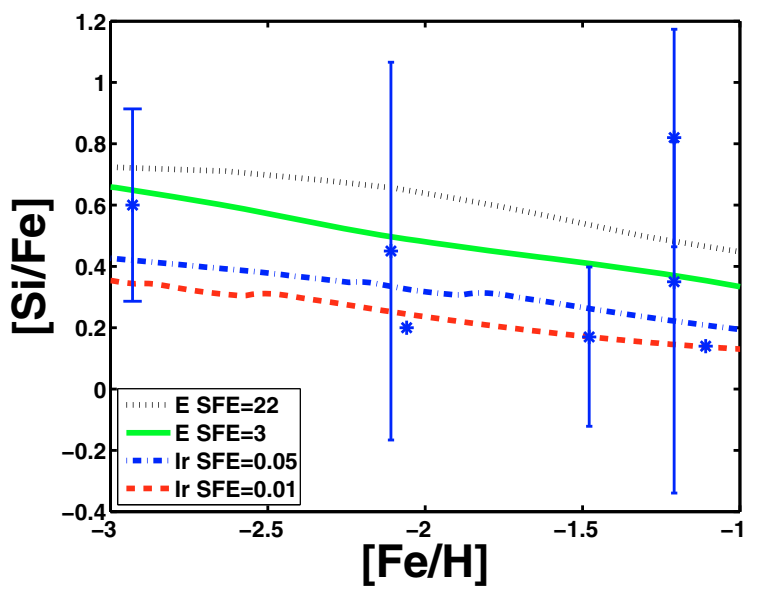

Fig. 6. $[\mathrm{Si} / \mathrm{Fe}]$ as function of $[\mathrm{Fe} / \mathrm{H}]$ of LGRB-DLA systems. The predictions are our from chemical evolution models of elliptical galaxies with $S F E=22$ (black dotted line) and $S F E=3$ (green solid line), and of irregular galaxies with $S F E=0.05$ (blue dashed-dotted line) and $S F E=0.01$ (red dashed line) (see text). Data are derived from Savaglio (2006) and Rau et al. (2010).

studied LGRBs are dwarf irregular galaxies in different evolutionary phases. In elliptical galaxies, there is no star formation after the galactic wind, therefore they can be rejected as hosts of LGRBs at low redshift. Since ellipticals are very rapidly enriched, by a fine-tuning of the formation redshift parameter, one can always find a model of elliptical capable of reproducing any subsolar $\log (\mathrm{X} / \mathrm{H})+12$ data at high redshift, but this would be an arbitrary assumption. We therefore need abundance ratios to test this situation (see Figs. 6 and 7 in the next section).

In Fig. 5, we show again the $\log (\mathrm{X} / \mathrm{H})+12$ versus redshift plot predicted by a model with $S F E=1.0$ but an infall mass of $10^{11} M_{\odot}$ that should represent the disk of a spiral galaxy such as the Milky Way (model 5). In this model, we also assumed that the gas accreted more slowly than dwarf irregulars. Detailed chemical evolution models for the Milky Way (e.g. Chiappini et al. 1997; Boissier \& Prantzos 1999; Hou et al. 2000; Chiappini et al. 2001; Yin et al. 2009) predict that the Milky Way disk formed in several Gyrs and inside-out. Here, we present a simplified one-zone model for a galactic disk that predicts average abundances, which helps to verify the behavior of the agemetallicity relation of a more massive galaxy with a higher SFE 


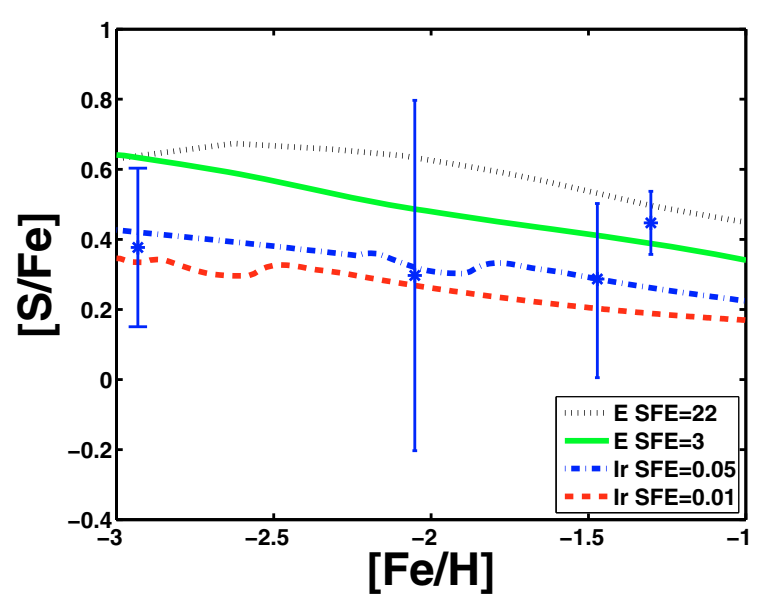

Fig. 7. $[\mathrm{S} / \mathrm{Fe}]$ as function of $[\mathrm{Fe} / \mathrm{H}]$ of LGRB-DLA systems. The predictions are from chemical evolution models for the elliptical galaxies with $S F E=22$ (black dotted line) and $S F E=3$ (green solid line), and for irregular galaxies with $S F E=0.05$ (blue dashed-dotted line) and $S F E=0.01$ (red dashed line) (see text). Data are derived from Savaglio (2006).

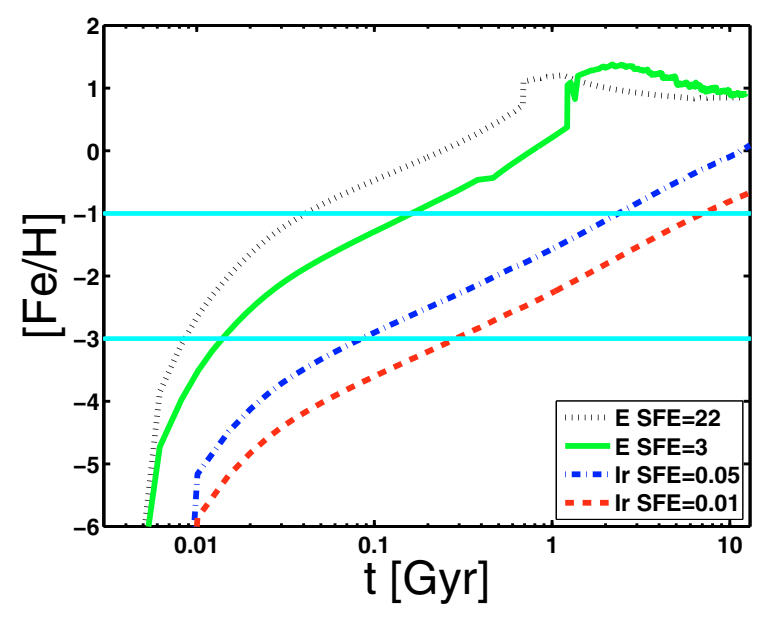

Fig. 8. Predicted $[\mathrm{Fe} / \mathrm{H}]$ versus galactic age for two irregular and two elliptical models, as indicated in the figure. The horizontal lines mark $[\mathrm{Fe} / \mathrm{H}]=-1.0$ and $[\mathrm{Fe} / \mathrm{H}]=-3.0$.

than typical irregulars. Figure 5 shows that this model, as happens for the model with infall mass $5 \times 10^{9} M_{\odot}$ and SFE typical of a spiral disk (model 1), cannot fit any of the LGRB hosts and therefore should be rejected. We note that we assumed a common redshift of galaxy formation for all the models $\left(z_{\mathrm{f}}=10\right)$ irrespective of the galaxy morphological type because old stars are in general found in every galaxy. If galaxies formed later, clearly they can not be the hosts of high-redshift LGRBs. However, irregulars may be the hosts of low-redshift LGRBs if they formed at redshift $z=1$, although in this case a slightly lower SFE would be required.

\subsection{Abundance ratios}

The abundance ratios of chemical elements, which have different timescales of production (such as $\alpha$-elements and Fe), can be used as useful criterion to study the star formation history in galaxies (see Matteucci 2001). In Figs. 6 and 7, we compare the $[\mathrm{Si} / \mathrm{Fe}]$ and $[\mathrm{S} / \mathrm{Fe}]$ ratios predicted by irregular and elliptical models with the available data for LGRB-DLAs. The data are

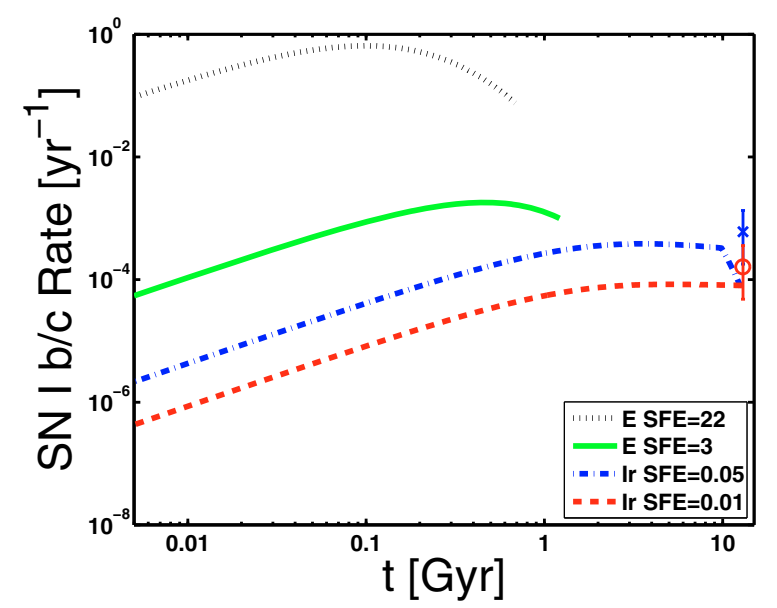

Fig. 9. Predicted $\mathrm{SNIb} / \mathrm{c}$ rate evolution as a function of galactic age for irregulars and ellipticals. The predictions are from our chemical evolution model of a elliptical galaxy assuming the star formation efficiency $S F E=22$ (black dotted line), $S F E=3$ (green solid line) and an irregular galaxy assuming $S F E=0.05$ (blue dashed-dotted line) and $S F E=0.01$ (red dashed line) (see text). Blue cross and red circle are the observed $\mathrm{SN} \mathrm{Ib/c}$ rates, derived by multiplying the $\mathrm{SN} \mathrm{Ib/c} \mathrm{rate} \mathrm{per}$ unit mass in irregular galaxies (Mannucci et al. 2005) by the predicted present stellar mass in each model.

derived from Savaglio (2006) and Rau et al. (2010). Although the error bars in the data are very large, the results imply that most of the data cannot be well fitted by the predictions of elliptical models. They are instead reproduced by the predictions of irregular models with a $S F E=0.05 \mathrm{Gyr}^{-1}$, thus confirming the results shown in Figs 1-4. However, more accurate data are necessary in the future to confirm this result.

In the case both of $\mathrm{Si}$ and $\mathrm{S}$, because of the large error bars in the observational data, the predictions of the elliptical models are marginally acceptable. In general, our predictions indicate that ellipticals, when forming stars (a passive evolutionary phase is of no interest because is without star formation and $\mathrm{SNe} \mathrm{Ib/c}$ are connected to star formation since they originate in massive stars), have higher $[\alpha / \mathrm{Fe}]$ ratios than spirals and irregulars and that their evolution is much shorter. Hence, if one measure were to the $[\alpha / \mathrm{Fe}]$ ratios of the gas in star-forming ellipticals at high redshift, one should find super-solar values. However, ellipticals are predicted to remain in a phase where their gas has a metallicity $[\mathrm{Fe} / \mathrm{H}]$ in common with those of DLAs (between -2.0 and -3.0$)$ for a very short time interval $(<0.1 \mathrm{Gyr})$, as shown in Fig. 8. Therefore, it is very difficult to observe these galaxies in this short time interval, another reason for excluding even small ellipticals from being DLA systems. If the DLAs did represent a particular evolutionary phase of a galaxy, regardless of morphological type, we should then observe DLAs with a wide range of properties (e.g. $[\alpha / \mathrm{Fe}])$, which reflecting the different star formation histories of galaxies of morphological type.

Since LGRBs are associated with $\mathrm{SNe} \mathrm{Ib} / \mathrm{c}$, we also computed these rates for different galaxies, and compared the predicted present-time $\mathrm{SN} \mathrm{Ib/c}$ rate with the observed one, as shown in Fig. 9. To compute this rate, we assumed that the progenitors of these SNe are either Wolf-Rayet stars with $M_{\text {in }}>25 M_{\odot}$ or massive binary systems in the mass range $12 M_{\odot} \leq M_{\text {in }} \leq$ $20 M_{\odot}$, as described in detail in Bissaldi et al. (2007). For the observational $\mathrm{SN} \mathrm{Ib/c} \mathrm{rate,} \mathrm{we} \mathrm{assumed} \mathrm{the} \mathrm{value} \mathrm{of} \mathrm{the} \mathrm{SN} \mathrm{Ib/c}$ rate per unit mass provided by Mannucci et al. (2005) for irregular galaxies, which is $0.54_{-0.38}^{+0.66} \mathrm{SNe}(100 \mathrm{yr})^{-1} 10^{-10} M_{\odot}$. We then multiplied this value by the predicted present-time stellar 
mass in each model (Table 1). The results show that our irregular models predict $\mathrm{SN} \mathrm{Ib} / \mathrm{c}$ rates in reasonable agreement with observations. In addition, the observed GRB redshift distribution peaks around $z=1$ (Fig. 1 in Savaglio et al. 2009) in reasonable agreement with our predicted peak of $\mathrm{SN} \mathrm{Ib} / \mathrm{c}$ rate (at $z=1$ corresponding to a galactic age $\sim 5$ Gyr, see Fig. 9). The sharp break in the $\mathrm{SN} \mathrm{Ib/c} \mathrm{rate} \mathrm{for} \mathrm{ellipticals} \mathrm{and} \mathrm{irregulars} \mathrm{with}$ $S F E=0.05$ occurs because at that time the galactic wind develops ( $S F E=0.01$ is too low to develop a wind). Gas is lost through the wind and the gas surface density decreases sharply (see Sect. 2 for details). As a consequence, the SFR drops and the $\mathrm{SN} \mathrm{Ib/c} \mathrm{rate} \mathrm{then} \mathrm{also} \mathrm{drops.}$

\section{Summary}

We have compared the data of LGRB-associated systems, LGRB-DLAs at high redshift, and LGRB host galaxies at low redshift, with chemical evolution models for galaxies of different morphological type. We have attempted to answer the following questions: i) which galaxies are the hosts of LGRBs? ii) are the LGRB systems part of an evolutionary sequence, in other words are they the same objects at low and high redshift as seen in different phases of their evolution?

Our conclusions can be summarized as follows:

1. If the observed high-redshift LGRB-DLAs and local LGRB host galaxies belonged to an evolutionary sequence, they should be irregulars with a common galaxy-formation redshift as high as $z_{\mathrm{f}}=10$, observed at different phases of their evolution. We can fit the majority of these systems using slowly evolving irregular models with a star formation efficiency, $S F E=0.1-0.05$. The adopted models were previously tested on local dwarf irregulars (see Yin et al. 2010a,b) and they may reproduce their properties.

2. We tested models with $S F E=1.0$, which resemble the evolution of the disk of the Milky Way (Chiappini et al. 1997; François et al. 2004; Cescutti et al. 2007). We considered the average properties of such a disk, and concluded that they fit neither high nor low redshift data since they predict too high absolute abundances. We cannot exclude, however, that they correspond to the outermost regions of spiral disks, since their properties are similar to those of irregulars (see Matteucci et al. 1997).

3. The rapid chemical enrichment at high redshift and subsequent passive evolution, following the occurrence of a galactic wind several Gyrs ago, of elliptical galaxies, suggests that they cannot be LGRB host galaxies at low redshift and that they are very unlikely hosts of LGRB-DLAs even at high redshift. The high observed $[\alpha / \mathrm{Fe}]$ ratios in ellipticals indicate in particular that most LGRB-DLAs can be neither a phase nor a part of ellipticals.

4. We have confirmed that the star formation history is the main driver of galaxy evolution. In particular, the properties of galaxies of different morphological type can be reproduced by simply changing the efficiency of star formation. By comparing the observed and predicted chemical properties of LGRB hosts, we have shown that they are most closely fitted by galaxy models with a lower SFE than either spirals or ellipticals, which is typical instead of irregular galaxies.

5. The elliptical models to which we compared the data refer to a very massive elliptical of infall mass $10^{12} M_{\odot}$, which is reproduced well by assuming a $S F E=22 \mathrm{Gyr}^{-1}$, and to a small elliptical of infall mass $10^{10} M_{\odot}$, which is reproduced well by assuming a lower $S F E=3 \mathrm{Gyr}^{-1}$. These assumptions reflect the downsizing in star formation that is necessary to reproduce the majority of the chemical and photometric properties of ellipticals. (e.g. downsizing in chemical enrichment, see Pipino \& Matteucci 2004, 2006, 2008). According to our anti-hierarchical galaxy formation scenario, we should with future data observe a mass-redshift relation for LGRB hosts.

Acknowledgements. Our thanks go to the anonymous referee for valuable comments and useful suggestions. J.Y. and F.M. acknowledge the financial support from PRIN2007 from Italian Ministry of Research, Prot. No. 2007JJC53X-001. J.Y. thanks the hospitality of the Department of Physics of the University of Trieste where this work was accomplished. J.Y. also thanks the financial support from the National Science Foundation of China No. 10573028, the Key Project No. 10833005, the Group Innovation Project No. 10821302, and 973 program No. 2007CB815402.

\section{References}

Akerman, C. J., Carigi, L., Nissen, P. E., Pettini, M., \& Asplund, M. 2004, A\&A, 414, 931

Bell, E. F., Naab, T., McIntosh, D. H., et al. 2006, ApJ, 640, 241

Bissaldi, E., Calura, F., Matteucci, F., Longo, F., \& Barbiellini, G. 2007, A\&A, 471,585

Boissier, S., \& Prantzos, N. 1999, MNRAS, 307, 857

Bradamante, F., Matteucci, F., \& D'Ercole, A. 1998, A\&A, 337, 338

Calura, F., \& Matteucci, F. 2006, ApJ, 652, 889

Calura, F., Matteucci, F., \& Vladilo, G. 2003, MNRAS, 340, 59

Calura, F., Dessauges-Zavadski, M., Prochaska, J. X., \& Matteucci, F. 2009, ApJ, 693, 1236

Campisi, M. A., De Lucia, G., Li, L., Mao, S., \& Kang, X. 2009, MNRAS, 400, 1613

Castro Cerón, J. M., Michałowski, M. J., Hjorth, J., et al. 2010, ApJ, 721, 1919

Cescutti, G., Matteucci, F., François, P., \& Chiappini, C. 2007, A\&A, 462, 943

Chary, R., Berger, E., \& Cowie, L. 2007, ApJ, 671, 272

Chen, X. Y., Liang, Y. C., Hammer, F., et al. 2010, A\&A, 515, A101

Chiappini, C., Matteucci, F., \& Gratton, R. 1997, ApJ, 477, 765

Chiappini, C., Matteucci, F., \& Romano, D. 2001, ApJ, 554, 1044

Chisari, N. E., Tissera, P. B., \& Pellizza, L. J. 2010, MNRAS, 408, 647

Cowie, L. L., Songaila, A., Hu, E. M., \& Cohen, J. G. 1996, AJ, 112, 839

François, P., Matteucci, F., Cayrel, R., et al. 2004, A\&A, 421, 613

Fynbo, J. P. U., Prochaska, J. X., Sommer-Larsen, J., Dessauges-Zavadsky, M., \& Møller, P. 2008, ApJ, 683, 321

Fynbo, J. P. U., Jakobsson, P., Prochaska, J. X., et al. 2009, ApJS, 185, 526

Galama, T. J., Vreeswijk, P. M., van Paradijs, J., et al. 1998, Nature, 395, 670

Graham, J. F., Fruchter, A. S., Kewley, L. J., et al. 2009, AIPCS, 1133, 269

Greiner, J., Krühler, T., Fynbo, J. P. U., et al. 2009, ApJ, 693, 1610

Hjorth, J., Sollerman, J., Møller, P., et al. 2003, Nature, 423, 847

Hou, J. L., Prantzos, N., \& Boissier, S. 2000, A\&A, 362, 921

Iwamoto, K., Brachwitz, F., Nomoto, K., et al. 1999, ApJS, 125, 439

Jakobsson, P., Fynbo, J. P. U., Ledoux, C., et al. 2006, A\&A, 460, L13

Kistler, M. D., Yüksel, H., Beacom, J. F., Hopkins, A. M., \& Wyithe, J. S. B. 2009, ApJ, 705, L104

Lamb, D. Q., \& Reichart, D. E. 2000, ApJ, 536, 1

Lanfranchi, G. A., \& Matteucci, F. 2003, MNRAS, 345, 71

Lapi, A., Kawakatu, N., Bosnjak, Z., et al. 2008, MNRAS, 386, 608

Levesque, E. M., Berger, E., Kewley, L. J., \& Bagley, M. M. 2010a, AJ, 139, 694

Levesque, E. M., Kewley, L. J., Graham, J. F., \& Fruchter, A. S. 2010b, ApJ, 712, L26

Li, A., Liang, S. L., Kann, D. A., et al. 2008, ApJ, 685, 1046

Liang, S. L., \& Li, A. 2009, ApJ, 690, L56

Mannucci, F., Della Valle, M., Panagia, N., et al. 2005, A\&A, 433, 807

Mao, J. 2010, ApJ, 717, 140

Matteucci, F. 2001, The chemical evolution of the Galaxy, Ap\&SS Library, 253

Matteucci, F., \& Padovani, P. 1993, ApJ, 419, 485

Matteucci, F., \& Pipino, A. 2002, ApJ, 569, L69

Matteucci, F., Molaro, P., \& Vladilo, G. 1997, A\&A, 321, 45

Matteucci, F., Ponzone, R., \& Gibson, B. K. 1998, A\&A, 335, 855

Michałowski, M. J., Hjorth, J., Castro Cerón, J. M., \& Watson, D. 2008, ApJ, 672,817

Møller, P., Warren, S. J., Fall, S. M., Fynbo, J. U., \& Jakobsen, P. 2002, ApJ, 574,51

Naab, T., \& Ostriker, J. P. 2006, MNRAS, 366, 899

Nuza, S. E., Tissera, P. B., Pellizza, L. J., et al. 2007, MNRAS, 375, 665

Padovani, P., \& Matteucci, F. 1993, ApJ, 416, 26 
Pettini, M. 2006, in The Fabulous Destiny of Galaxies: Bridging Past and Present, ed. V. Le Brun, A. Mazure, S. Arnouts, \& D. Burgarella, 319

Pian, E., Mazzali, P. A., Masetti, N., et al. 2006, Nature, 442, 1011

Pipino, A., \& Matteucci, F. 2004, MNRAS, 347, 968

Pipino, A., \& Matteucci, F. 2006, MNRAS, 365, 1114

Pipino, A., \& Matteucci, F. 2008, A\&A, 486, 763

Pipino, A., Fan, X., Matteucci, F., et al. 2010, A\&A, accepted [arXiv: 1008.3875$]$

Pontzen, A., Deason, A., Governato, F., et al. 2010, MNRAS, 402, 1523

Prochaska, J. X., Chen, H., Bloom, J. S., et al. 2007a, ApJS, 168, 231

Prochaska, J. X., Chen, H., Dessauges-Zavadsky, M., \& Bloom, J. S. 2007b, ApJ, 666, 267

Rau, A., Savaglio, S., Krühler, T., et al. 2010, ApJ, 720, 862

Romano, D., Matteucci, F., Salucci, P., \& Chiappini, C. 2000, ApJ, 539, 235

Romano, D., Tosi, M., \& Matteucci, F. 2006, MNRAS, 365, 759

Salpeter, E. E. 1955, ApJ, 121, 161

Salvaterra, R., Della Valle, M., Campana, S., et al. 2009, Nature, 461, 1258

Savaglio, S. 2006, New J. Phys., 8, 195

Savaglio, S., Glazebrook, K., \& Le Borgne, D. 2009, ApJ, 691, 182

Schmidt, M. 1959, ApJ, 129, 243
Shapley, A. E., Steidel, C. C., Erb, D. K., et al. 2005, ApJ, 626, 698

Svensson, K. M., Levan, A. J., Tanvir, N. R., Fruchter, A. S., \& Strolger, L. 2010, MNRAS, 405, 57

Tanvir, N. R., Fox, D. B., Levan, A. J., et al. 2009, Nature, 461, 1254

van den Hoek, L. B., \& Groenewegen, M. A. T. 1997, A\&AS, 123, 305

Wolf, C., \& Podsiadlowski, P. 2007, MNRAS, 375, 1049

Wolfe, A. M. 2005, in Probing Galaxies through Quasar Absorption Lines, ed. P. Williams, C.-G. Shu, \& B. Menard, IAU Colloq., 199, 148

Wolfe, A. M., Turnshek, D. A., Smith, H. E., \& Cohen, R. D. 1986, ApJS, 61, 249

Woosley, S. E. 1993, ApJ, 405, 273

Woosley, S. E., \& Heger, A. 2006a, ApJ, 637, 914

Woosley, S. E., \& Heger, A. 2006b, AIPCS, 836, 398

Yin, J., Hou, J. L., Prantzos, N., et al. 2009, A\&A, 505, 497

Yin, J., Magrini, L., Matteucci, F., et al. 2010a, A\&A, 520, A55

Yin, J., Matteucci, F., \& Vladilo, G. 2010b, A\&A, submitted

Yoon, S., Langer, N., Cantiello, M., Woosley, S. E., \& Glatzmaier, G. A. 2008, IAUS, 250, 231

Zwaan, M. A., van der Hulst, J. M., Briggs, F. H., Verheijen, M. A. W., \& Ryan-Weber, E. V. 2005, MNRAS, 364, 1467 